\title{
New Insights into the Nature of Cerebellar-Dependent Eyeblink Conditioning Deficits in Schizophrenia: A Hierarchical Linear Modeling Approach
}

\author{
Amanda R. Bolbecker, Isaac T. Petersen, Jerillyn S. Kent, Josselyn M. Howell, \\ Brian F. O'Donnell and William P. Hetrick*
}

Department of Psychological and Brain Sciences, Indiana University, Bloomington, IN, USA

OPEN ACCESS

Edited by:

Tracy L. Greer,

University of Texas Southwestern

Medical Center, USA

Reviewed by:

Jeffrey Varner,

Purdue University, USA

Litao Sun,

The Scripps Research Institute, USA

Eleftheria Pervolaraki,

University of Leeds, UK

Michael Killian,

University of Texas at Arlington, USA

${ }^{*}$ Correspondence:

William P. Hetrick

whetrick@indiana.edu

Specialty section:

This article was submitted to

Systems Biology,

a section of the journal

Frontiers in Psychiatry

Received: 05 June 2015 Accepted: 11 January 2016 Published: 25 January 2016

Citation:

Bolbecker AR, Petersen IT, Kent JS,

Howell JM, O'Donnell BF and Hetrick WP (2016) New Insights into the Nature of Cerebellar-Dependent

Eyeblink Conditioning Deficits in Schizophrenia: A Hierarchical Linear

Modeling Approach.

Front. Psychiatry 7:4.

doi: 10.3389/fpsyt.2016.00004
Evidence of cerebellar dysfunction in schizophrenia has mounted over the past several decades, emerging from neuroimaging, neuropathological, and behavioral studies. Consistent with these findings, cerebellar-dependent delay eyeblink conditioning (dEBC) deficits have been identified in schizophrenia. While repeated-measures analysis of variance is traditionally used to analyze dEBC data, hierarchical linear modeling (HLM) more reliably describes change over time by accounting for the dependence in repeated-measures data. This analysis approach is well suited to dEBC data analysis because it has less restrictive assumptions and allows unequal variances. The current study examined dEBC measured with electromyography in a single-cue tone paradigm in an age-matched sample of schizophrenia participants and healthy controls ( $N=56$ per group) using HLM. Subjects participated in 90 trials (10 blocks) of dEBC, during which a $400 \mathrm{~ms}$ tone co-terminated with a $50 \mathrm{~ms}$ air puff delivered to the left eye. Each block also contained 1 tone-alone trial. The resulting block averages of dEBC data were fitted to a three-parameter logistic model in HLM, revealing significant differences between schizophrenia and control groups on asymptote and inflection point, but not slope. These findings suggest that while the learning rate is not significantly different compared to controls, associative learning begins to level off later and a lower ultimate level of associative learning is achieved in schizophrenia. Given the large sample size in the present study, HLM may provide a more nuanced and definitive analysis of differences between schizophrenia and controls on dEBC.

Keywords: schizophrenia, eyeblink conditioning, cerebellum, associative learning, reflex conditioning, conditioned response, cognition, psychosis

\section{INTRODUCTION}

Schizophrenia is a complex disorder with diverse symptoms and heterogeneous expression. Besides its cardinal psychotic symptoms, cognitive and motor abnormalities are prominent symptoms of the disorder. The cognitive dysmetria theory of schizophrenia (1) provides a unitary framework that can account for the disparate symptoms of schizophrenia. It posits that disruptions in the 
cortico-cerebello-thalamo-cortical circuit (CCTCC) lead to poor coordination of information, resulting in different symptom constellations. Given that the cerebellum plays a role in temporal processing (2), it may occupy a unique role in this circuit by modulating the temporal coordination of information. Consistent with this proposition, evidence collected over the last several decades points to not only an important cerebellar role in coordinated movement and motor learning, but also non-motor psychological processes, most notably cognition (3-8). The neuroanatomical substrate for these functional effects has been revealed by studies confirming that the $\mathrm{CB}$ is reciprocally connected to prefrontal, parietal, and motor/premotor cortex (9-12). It is not surprising then that lesions to the cerebellum can produce symptoms commonly seen in schizophrenia, including visuospatial deficits, attention deficits, executive dysfunction, flattened affect, disinhibited, and socially inappropriate behavior (6).

Neuropathological and neuroimaging studies have documented morphological and functional cerebellar abnormalities in schizophrenia. For example, subjects with schizophrenia have reduced bilateral cerebellar volume (13), abnormal cerebellar connectivity to cerebral regions involved in both motor and cognitive functions (14), cerebellar morphological abnormalities (15), and reductions in Purkinje cell size and density (16-18). Even groups at clinical and familial risk for psychosis show reduced cerebellar gray matter (19) compared to non-risk groups. However, negative findings exist both in the neuroimaging (20) and neuropathology (21) literature.

Importantly, first-episode (22-24) and antipsychotic medication naïve schizophrenia patients (25) have reduced cerebellar volume, suggesting that cerebellar abnormalities are characteristic of the disorder rather than medication use. Perhaps most convincingly, cerebellar volume is associated with cognitive deficits (26) as well as symptoms of depression, negative symptoms, and psychotic features in schizophrenia $(25,27,28)$, suggesting that illness severity or progression may coincide with cerebellar degradation.

Delay eyeblink conditioning ( $\mathrm{dEBC})$ is an associative learning task that is highly dependent upon cerebellar functioning (29-31), in. The neuro-circuitry of this task has been extensively studied, and evidence overwhelmingly supports the conclusion that the cerebellum is critical both for learning the association between the unconditioned and conditioned stimuli and for the expression of the conditioned eyeblink response $(32,33)$. Numerous additional brain regions (i.e., hippocampus, medial septum, frontal cortex) can change the way in which the eyeblink response is expressed (34), neuroplasticity in the cerebellum initially elicits the classically conditioned eyeblink response (35).

Over the past decade, accumulating evidence indicates that cerebellar-mediated $\mathrm{dEBC}$ associative learning is abnormal in schizophrenia (36-38), schizotypal personality disorder (39), and first-degree relatives of schizophrenia patients (38). These associative learning deficits in schizophrenia may be remediated by pharmacological intervention (37).

One outstanding issue in the dEBC literature is statistical in nature. Specifically, a repeated-measures analysis of variance (ANOVA) is commonly used to analyze dEBC data, despite the availability of superior and more sophisticated statistical techniques, such as hierarchical linear modeling (HLM), which may reveal more reliable and nuanced findings. In our previous studies of AEBC using ANOVA, we have found conflicting results with respect to whether the learning rate (e.g., the block by group interaction in ANOVA) differs between groups. Several studies have found that the schizophrenia group had a reduced acquisition rate $(36,39)$, while others found no difference between groups $(38,40)$. Notably, the study with the largest sample size $(N=62)$ found a reduced average percentage of conditioned responses from subjects with schizophrenia, but no between-group differences in acquisition rate compared to healthy controls (40).

Hierarchical linear modeling is particularly well suited to $\mathrm{dEBC}$ data analysis and is superior to repeated-measures ANOVA for measuring time-dependent change because it takes into consideration the statistical dependencies in repeated-measures designs. HLM can be considered a special case of regression that can accommodate variance on more than one level (i.e., nested data), in this case, at both the individual level and at the group level. In HLM, the best-fitting line for each individual is identified, but each line fit is also influenced by the trajectories of other group members. This aspect of HLM has the effect of increasing the accuracy of each individual's fit while minimizing the error of measurement at the individual and group level. Moreover, HLM has less restrictive assumptions, can tolerate missing data points, and can accommodate hierarchical or nested data structures (41). Perhaps the greatest strength of HLM is that heterogeneity of variance is treated as potentially meaningful information that can help to identify significant interactions between variables (42), whereas in ANOVA it is treated as a nuisance factor. Finally, HLM can be used to examine growth curves that model traditional learning curves so that important parameters, such as the slope, asymptote, and inflection point of the fitted curves can be quantified. [For a more comprehensive explanation of the use of HLM in repeated-measures designs, please see Ref. (43)].

Hierarchical linear modeling was implemented in a recent study $(44,45)$ in which $\mathrm{dEBC}$ data from healthy controls, individuals with schizophrenia, and first-degree relatives of individuals with schizophrenia ( $N=18$ per group) were fitted to a linear model. Differences in acquisition rate (i.e., slope), indicating a slower rate of associative learning was found between both the schizophrenia and family members groups compared to controls. In the present study, data from a larger schizophrenia sample was age-matched to controls $(N=59$ per group) and HLM was applied to a three-parameter logistic growth model to more closely approximate a learning curve. We predicted that the slope of the learning curve would be lower for the schizophrenia group, indicating a slower learning rate. We also expected that the asymptote - the maximum level of performance - would be lower in schizophrenia, and that the inflection point, which is the point on the learning curve when learning begins to slow down and level off, would occur later.

\section{MATERIALS AND METHODS}

\section{Participants}

Participants were 56 individuals ( 17 females) who were diagnosed with schizophrenia and 56 age-matched control participants (29 
females). Control participants had no history of psychotic and mood disorders and no history of schizophrenia spectrum disorders within first-degree relatives. Data from 36 individuals with schizophrenia (12 females) and 32 controls ( 15 females) included in this study had been included in an earlier study of dEBC that used more traditional analysis methods (40). Participants with schizophrenia were recruited through outpatient and inpatient units at local hospitals. The control group was recruited by posting community and newspaper advertisements. Participants' demographic, clinical, and medication information can be seen in Table 1. Welch's $t$-test showed that, as expected due to agematching, the mean age of schizophrenia participants did not differ from controls $[t(1,112)=-0.29, P=0.77]$. Sex was significantly different across groups $[\chi 2(1)=4.46, P=0.035]$, with more males in the schizophrenia group (see Table 1). Importantly, sex was used as a covariate in the HLM analyses and it did not significantly improve model fit $(p>0.05)$.

The Diagnostic and Statistical Manual of Mental Disorders-IV Axis I Disorders (SCID-I) (46) sections for mood disorders, psychotic disorders, and substance abuse disorders was used to diagnose participants in the schizophrenia group. Medical records were consulted to refine diagnoses when necessary. The non-patient version of SCID-I (47) sections for mood, psychotic, and substance abuse, as well as the SCID II, was used to identify controls without a history of psychiatric or personality disorders. The positive and negative syndrome scale (PANSS) (48) was used to rate clinical symptoms in the schizophrenia group. A total of 53 of the 56 participants in the schizophrenia group had PANSS scores available within 2 weeks of the time of dEBC testing.

Participants were excluded from the experiment if they had clinically significant hearing loss, cardiovascular disease, an intelligence quotient (IQ) score of less than 70, had received electroconvulsive therapy, or if they had a history of neurological disorders, head injury resulting in loss of consciousness, or alcohol or substance dependence within the 3 months prior to their participation in the experiment. Additional exclusion criteria for potential control group participants were history of psychotic or mood disorders, or having a first-degree relative

TABLE 1 | Demographic, clinical, and medication information.

\begin{tabular}{lcc} 
& Schizophrenia & Controls \\
\hline Age (years) & $M=36.4(\mathrm{SD}=10)$ & $M=35.8(\mathrm{SD}=10)$ \\
Sex (M:F) & $39: 17$ & $27: 29$ \\
PANSS total score & $M=59(\mathrm{SD}=13)$ & - \\
$\quad$ Positive & $M=16(\mathrm{SD}=6)$ & - \\
$\quad$ Negative & $M=15(\mathrm{SD}=5)$ & - \\
General & $M=28(\mathrm{SD}=6)$ & 0 \\
aPast alcohol dependence & 13 & 0 \\
Past illicit drug dependence & 16 & 56 \\
bPsychotropic medication & & 0 \\
$\quad$ No antipsychotic medication & 6 & 0 \\
Atypical antipsychotic & 44 & \\
Typical antipsychotic & 12 &
\end{tabular}

aNine schizophrenia patients met criteria for both past alcohol and other drug dependence.

${ }^{b}$ Eight schizophrenia patients were taking both typical and atypical antipsychotic drugs at the time of testing. Medication information was not available for two participants with schizophrenia. with a schizophrenia spectrum diagnosis. All aspects of this study were approved by the Indiana University Human Subjects Institutional Review Board (IUB-IRB; Protocol \#1009001702), and all participants provided written informed consent prior to participation in the study.

\section{Delay Eyeblink Conditioning Procedure}

The experiment consisted of 10 blocks of dEBC, with 10 trials per block. Of these 10 trials, 9 were paired with a conditioned stimulus tone lasting $400 \mathrm{~ms}(1000 \mathrm{~Hz}, 80 \mathrm{~dB})$ that co-terminated with a $50 \mathrm{~ms}$ unconditioned stimulus air puff $(10 \mathrm{psi}$ at the source). A single tone-alone trial was also randomly presented during each block. The experiment began with eight unconditioned stimuli (15 s average inter-trial interval with a range of $10-20 \mathrm{~s}$ ) that were presented alone to assess the integrity of eyeblink responses. Participants rated neutral pictures from the International Affective Picture System (49) throughout the experiment to maintain alertness. Pictures were presented for $2 \mathrm{~s}$ between trials and participants indicated the pleasantness of each picture on a response pad. Participants were monitored using a closed circuit camera to ensure their eyes remained open during the experiment. In cases in which a participant's eyes appeared to close, the experiment was briefly suspended so alertness could be re-established by turning on the lights and offering the participant a drink of water.

\section{Procedure}

Electromyographic activity was recorded from the orbicularis palpebrarum of the left eye by placing two bipolar electrodes $1 \mathrm{~cm}$ below the left eyelid, approximately $1 \mathrm{~cm}$ apart, and centered beneath the pupil. A ground electrode was placed on the forehead. The $50 \mathrm{~ms}$ unconditioned stimulus air puff was delivered to the left eye via copper tubing affixed to lens-less glasses and connected to plastic tubing (approximately 120") connected to a regulator. Ear inserts (E-A-RLINK - Aearo Company Auditory Systems) were used to deliver the conditioned stimulus tone. Electromyographic recordings were continuously recorded $(2.5 \mathrm{kHz}$ A/D rate; highpass filter $=1 \mathrm{~Hz}$; low-pass filter $=500 \mathrm{~Hz}$; gain $=1000)$ and stored offline for further processing.

\section{Data Processing}

The continuous dEBC data files were segmented into $1086 \mathrm{~ms}$ epochs starting $500 \mathrm{~ms}$ before the conditioned stimulus onset. Data were high-pass filtered using a $28 \mathrm{~Hz}$ ( $6 \mathrm{~dB}$ per octave) filter, rectified, then smoothed using a 41 point Gaussian weighted moving average. The 90 paired dEBC trials from each experiment were analyzed using DataMunch, a MatLab program specifically designed for eyeblink conditioning data analysis (36, 38-40, 44, $45,50-52$ ). Blinks that occurred between 25 and $100 \mathrm{~ms}$ were characterized as alpha responses, which occur in response to the conditioned response tone onset and are reflexive, orienting responses that are not learning-related phenomena. For each participant, eyeblinks were counted as conditioned responses if they exceeded 5 SDs of baseline activity (baseline $=125 \mathrm{~ms}$ prior to conditioned stimulus onset) for each trial.

Trials in which electromyographic activity increased during the time window beginning $25 \mathrm{~ms}$ prior to the conditioned 
stimulus onset through $75 \mathrm{~ms}$ post-onset were excluded from analysis. These trials were excluded because blinks during this interval are not considered learning-related, and can interfere with the emission of a true conditioned response eyeblink.

Conditioned responses were recorded when an eyeblink occurred between 100 and $350 \mathrm{~ms}$ after the tone's onset, the time interval corresponding to the $250 \mathrm{~ms}$ prior to the unconditioned stimulus onset. The onset latency was calculated as the time when the electromyographic activity exceeded 0.5 SDs from baseline activity.

\section{Statistical Analysis}

Block-by-block percentages of conditioned responses from $\mathrm{dEBC}$ experiments were fitted to growth curve models using HLM. Conditioned response averages for each of the 10 blocks for each individual were calculated and the best-fitting line was generated, resulting in one line for each participant - a total of 154 lines. Eleven from this initial group (six participants with schizophrenia and five controls) were dropped from the analysis because they failed to exhibit conditioned responding such that the difference between the last and the first estimation of a linear curve fit was $<0 \%$. Therefore, 143 participants remained for age-matching (60 in the schizophrenia group; 83 in the control group). The final sample included 59 participants with schizophrenia who were age-matched to a healthy control whose age was within 2 years of their own.

The lme function of the nlme package (53) in R 3.0 ( $R$ Development Core Team, 2009) was used to model associative learning for growth curve modeling in HLM. Models used maximum likelihood estimation, except when testing whether effects should be fixed or random, in which case restricted maximum likelihood was used as suggested by Singer and Willett (54). Linear and non-linear forms of change were examined with nested model comparisons using the likelihood ratio test. Model fit was examined with pseudo- $R^{2}(54)$, which was calculated by the squared correlation between the model's fitted and observed values, representing the proportion of variance in the outcome explained by the model.

A three-parameter logistic growth curve with a randomly varying asymptote and fixed values for the slope and inflection point was used, which fit the data well (pseudo- $R^{2}=0.73$ ). The model allowed different asymptote estimates across participants but not different estimates of slope or inflection point (but were allowed to differ by group). A random effect of asymptote was a better model fit than a model with a random effect of inflection point, and models with a random effect of slope did not converge. For each individual, logistic growth curves were fit to associative learning curves across the 10 blocks of the experiment. These logistic curves estimated whether the groups were different for each of the three parameters: slope, inflection point, and asymptote. The inflection point is the point on the curve where it changes curvature, and the asymptote is where learning begins to level off. The slope measures the change in associative learning over time and was used to assess differences in learning rate between groups.

We attempted to analyze data from conditioned response onset latency, but the data fit a logistic growth curve model poorly (pseudo- $R^{2}=0.24$ ). Therefore, although all indications were that no differences on primary dependent variables could be observed, given the lack of fit and consequent unreliability of statistical measures, we have not included this analysis in the Section "Results."

Using three separate statistical tests of between-group differences (schizophrenia vs. controls for asymptote, slope, and inflection point), a Bonferroni-corrected alpha level of $P<0.017$ ( $P<0.05 / 3$ comparisons $)$ was deemed significant, although results with $P<0.05$ are reported.

\section{RESULTS}

\section{Baseline Unconditioned Response Amplitude}

Differences in conditioned response measurements could arise from impairment in general eyeblink performance. Therefore, to ensure that any observed differences between groups on the percentage of conditioned responses was not due to such a general performance issue, eight unconditioned stimulus air puffs were presented alone at the beginning of the experiment. Baseline unconditioned response amplitude was available for a total of 41 participants with schizophrenia and 42 controls. Neither the average peak unconditioned response amplitudes $[F(1,81)=3.17, P=0.08]$ nor latencies $[F(1,81)=0.003, P=0.96]$ were significantly different between groups. While the differences in amplitude did not reach significance, it is important to note that average group differences indicated that the schizophrenia group had larger unconditioned response amplitudes $(M=97.89 \mu \mathrm{V}$, $\mathrm{SD}=23.27)$ compared to controls $(M=89.64 \mu \mathrm{V}, \mathrm{SD}=18.79)$. This finding is consistent with earlier findings that unconditioned response amplitude was larger on paired $\mathrm{AEBC}$ trials in schizophrenia (40). Overall, these findings suggest that differences in conditioned responses are unlikely to be due to deficits in blink performance in the schizophrenia group.

\section{Percentage of Conditioned Responses}

Parameter estimates of the logistic model examining learning curves of the percentage of conditioned responses are in Table 2. Figure 1 shows the line fits for each participant, the group average fitted line, and the conditioned response average for each of the 10 blocks. Findings suggest that the difference in learning between the beginning and end of the experiment is similar between groups,

TABLE 2 | Parameter estimates for the HLM growth curve model for percentage of conditioned responses.

\begin{tabular}{lrrrr}
\hline & Value (SE) & DF & $\boldsymbol{t}$-value & $\boldsymbol{p}$-value \\
\hline$R^{2}=0.73$ & & & & \\
Asymptote & $68.92(3.46)$ & 1003 & -19.91 & 0.000 \\
$\quad$ SZ-HC & $-20.31(4.97)$ & 1003 & -4.09 & $0.000^{\star}$ \\
Inflection Point & $0.64(0.17)$ & 1003 & 3.59 & 0.000 \\
SZ-HC & $0.74(0.27)$ & 1003 & 2.77 & $0.006^{\star}$ \\
Slope & $1.1(0.19)$ & 1003 & 5.88 & 0.000 \\
$\quad$ SZ-HC & $0.51(0.33)$ & 1003 & 1.59 & 0.112 \\
\hline
\end{tabular}

SZ, schizophrenia, HC, healthy controls.

*Indicates differences between groups with a significance at $P<0.017$. 


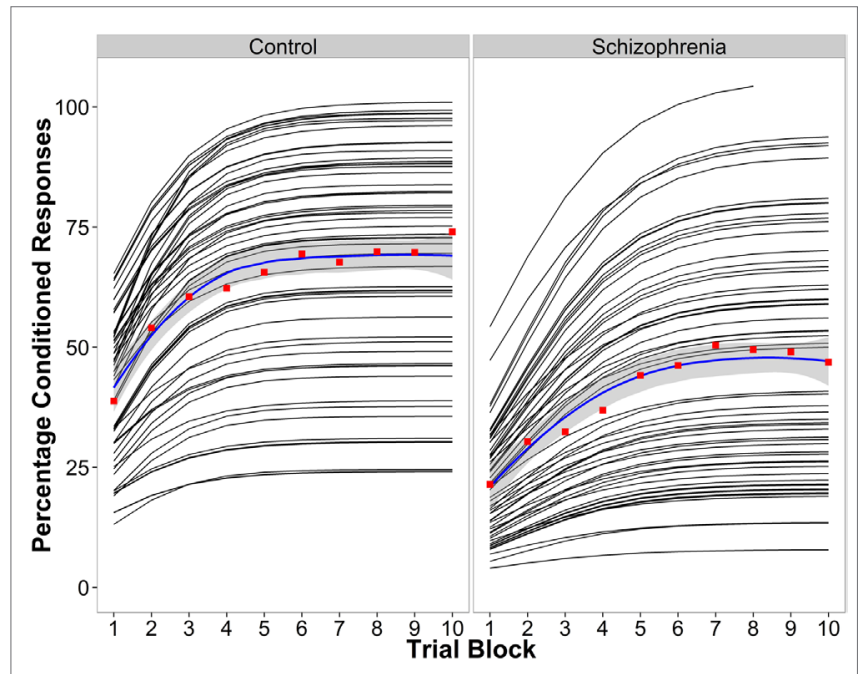

FIGURE 1 | Conditioned response data for the control group (left) and the schizophrenia group (right). The logistic curve fit for each individual (black lines), the average percentage of CRs for the raw data for each block in red, and the group average logistic curve fit in blue.

but that learning saturates later in the schizophrenia group, and the level at which saturation occurs is lower in the schizophrenia group. When the groups were considered together, performance improved across the 10 blocks of the experiment, $t(1003)=5.88$, $P<0.001, \mathrm{SE}=0.19$, and the rate of learning did not differ between groups, $t(1003)=1.59, P=0.11, \mathrm{SE}=0.33$. However, the asymptote was significantly lower in the schizophrenia group, $t(1003)=-4.09, P<0.001, \mathrm{SE}=4.97$. Moreover, the inflection point occurred later in schizophrenia group $[t(1003)=2.77$, $P=0.006, \mathrm{SE}=0.27]$. These results indicate that the rate of learning over the course of the experiment (the slope), measured as the difference between blocks 1 and 10 on the fitted logistic curves, was not significantly different between groups. However, the reduced asymptote in schizophrenia makes the slope more similar between groups even though the inflection point occurred later. Overall, the schizophrenia group attained a lower ultimate level of learning and took longer to achieve this maximum.

\section{Correlations with Clinical Symptoms}

We examined associations of participants' estimates on each of the three logistic model parameters for the percentage of conditioned responses with PANSS positive, negative, general, and total scores using bivariate correlations with age partialed out. There were no significant correlations between any behavioral parameters and clinical variables.

\section{DISCUSSION}

The goal of the present study was to extend and clarify results of earlier studies examining $\mathrm{dEBC}$ in schizophrenia using more sophisticated statistical models. HLM of data fitted to a logistic growth curve model provided insight into how three components of the learning curve change over time in schizophrenia. Overall, associative learning in the schizophrenia group leveled off at a lower level compared to controls, and took longer to reach the maximal learning level. Surprisingly, the rate of learning (i.e., slope) within subjects with schizophrenia was not significantly different from controls.

Analysis of dEBC data using HLM, a superior analytic approach compared to ANOVA, suggests robust differences between subjects in the control and schizophrenia groups. Cerebellar abnormalities in schizophrenia are most likely responsible for these behavioral $\mathrm{dEBC}$ differences. The regions of cerebellar cortex that show reduced regional cerebral blood flow ( $\mathrm{rCBF}$ ) during $\mathrm{dEBC}$ in unmedicated schizophrenia (55) also overlap with those identified as fundamental to normal expression of conditioned eyeblink responses in animal studies (56-59). The interpositus nucleus is necessary for the acquisition and retention of the conditioned eyeblink response with cerebellar cortical sites, in particular long-term depression at the parallel fiber-Purkinje cell synapse, modulating important aspects of the gain and timing of the response (see, Ref. (60) for extensive review). Human studies of populations with cerebellar lesions or degeneration largely support these findings, and also suggest that purely cortical lesions produce significant reductions in the expression of conditioned responses, but do not abolish them (61). Importantly, cerebellar cortical structure is associated with conditioned response timing (62) and acquisition (63). Taken together, these findings suggest that abnormalities in the interpositus nuclei and the cortex of the cerebellum contribute to the dEBC deficits observed in schizophrenia.

Our laboratory has undertaken a program of research that aims to tackle outstanding questions about cerebellar abnormalities in schizophrenia. We have previously reported deficits in schizophrenia on timing tasks that rely heavily on cerebellar-based timing mechanisms, including paced finger-tapping (64) and a temporal bisection task $(45,65,66)$. Using neuroimaging techniques, we can more definitively understand the extent to which the dEBC deficits in schizophrenia are uniquely attributable to alterations in cerebellar function compared to other cortical and subcortical circuits in which the cerebellum participates. We are currently using functional magnetic resonance imaging in conjunction with $\mathrm{AEBC}$ and paced finger-tapping to determine how cerebellar functional and structural abnormalities contribute to performance deficits in schizophrenia. Moreover, our recent studies have identified dEBC abnormalities in an intermediate phenotype of schizophrenia, namely schizotypal personality disorder (39), and in first-degree relatives of individuals with schizophrenia (44), suggesting that $\mathrm{AEBC}$ impairments may be risk markers for schizophrenia. Ongoing studies of first-degree relatives will determine whether familial risk is associated with morphological and functional alterations in the cerebellum and related circuits.

Our current studies and others addressing similar questions may provide evidence that the cerebellum is a potential therapeutic target for remediating symptoms of schizophrenia. Indeed, preliminary evidence supports this idea. For example, secretin is a neuropeptide with receptors in the cerebellum, which permitted us to make predictions based on a mechanistic model of its actions within the cerebellar cortex $(67,68)$. When we administered secretin to a small group of participants with schizophrenia, it significantly improved $\mathrm{AEBC}$ performance and validated the utility of the cerebellum as a potential pharmacological target 
(37) [c.f., Ref. $(69,70)]$. Similarly, a small sample of individuals with treatment-resistant schizophrenia underwent theta-burst transcranial magnetic stimulation of the cerebellum and experienced both improved mood symptoms and enhanced cognitive performance (71). Taken together, efforts to identify cerebellardependent biomarkers will facilitate the development of new potential therapeutic targets within the cerebellum that could provide previously unexplored avenues of treatment that are sorely needed for this perplexing disorder.

\section{REFERENCES}

1. Andreasen NC. A unitary model of schizophrenia: Bleuler's "fragmented phrene" as schizencephaly. Arch Gen Psychiatry (1999) 56(9):781-7. doi:10.1001/archpsyc.56.9.781

2. Ivry RB, Spencer RM. The neural representation of time. Curr Opin Neurobiol (2004) 14(2):225-32. doi:10.1016/j.conb.2004.03.013

3. Ivry RB, Keele SW. Timing functions of the cerebellum. J Cogn Neurosci (1989) 1(2):136-52. doi:10.1162/jocn.1989.1.2.136

4. Katz DB, Steinmetz JE. Psychological functions of the cerebellum. Behav Cogn Neurosci Rev (2002) 1:229-41. doi:10.1177/1534582302001003004

5. Leiner HC, Leiner AL, Dow RS. The human cerebro-cerebellar system: its computing, cognitive, and language skills. Behav Brain Res (1991) 44(2):113-28. doi:10.1016/S0166-4328(05)80016-6

6. Schmahmann JD. Disorders of the cerebellum: ataxia, dysmetria of thought, and the cerebellar cognitive affective syndrome. J Neuropsychiatry Clin Neurosci (2004) 16(3):367-78. doi:10.1176/appi.neuropsych.16.3.367

7. Schmahmann JD, Sherman JC. Cerebellar cognitive affective syndrome. Int Rev Neurobiol (1997) 41:433-40. doi:10.1016/S0074-7742(08)60363-3

8. Schmahmann JD, Sherman JC. The cerebellar cognitive affective syndrome. Brain (1998) 121(Pt 4):561-79. doi:10.1093/brain/121.4.561

9. Clower DM, West RA, Lynch JC, Strick PL. The inferior parietal lobule is the target of output from the superior colliculus, hippocampus, and cerebellum. $J$ Neurosci (2001) 21(16):6283-91.

10. Middleton FA, Strick PL. Anatomical evidence for cerebellar and basal ganglia involvement in higher cognitive function. Science (1994) 266(5184):458-61. doi:10.1126/science.7939688

11. Middleton FA, Strick PL. Cerebellar output: motor and cognitive channels. Trends Cogn Sci (1998) 2(9):348-54. doi:10.1016/S1364-6613(98)01220-0

12. Middleton FA, Strick PL. Cerebellar projections to the prefrontal cortex of the primate. J Neurosci (2001) 21(2):700-12.

13. Laidi C, d'Albis MA, Wessa M, Linke J, Phillips ML, Delavest M, et al. Cerebellar volume in schizophrenia and bipolar I disorder with and without psychotic features. Acta Psychiatr Scand (2015) 131(3):223-33. doi:10.1111/ acps. 12363

14. Shinn AK, Baker JT, Lewandowski KE, Ongur D, Cohen BM. Aberrant cerebellar connectivity in motor and association networks in schizophrenia. Front Hum Neurosci (2015) 9:134. doi:10.3389/fnhum.2015.00134

15. Schmitt A, Schulenberg W, Bernstein HG, Steiner J, Schneider-Axmann $\mathrm{T}$, Yeganeh-Doost $\mathrm{P}$, et al. Reduction of gyrification index in the cerebellar vermis in schizophrenia: a post-mortem study. World J Biol Psychiatry (2011) 12(Suppl 1):99-103. doi:10.3109/15622975.2011.598379

16. Maloku E, Covelo IR, Hanbauer I, Guidotti A, Kadriu B, Hu Q, et al. Lower number of cerebellar Purkinje neurons in psychosis is associated with reduced reelin expression. Proc Natl Acad Sci U S A (2010) 107(9):4407-11. doi:10.1073/pnas.0914483107

17. Reyes MG, Gordon A. Cerebellar vermis in schizophrenia. Lancet (1981) 2(8248):700-1. doi:10.1016/S0140-6736(81)91039-4

18. Tran KD, Smutzer GS, Doty RL, Arnold SE. Reduced Purkinje cell size in the cerebellar vermis of elderly patients with schizophrenia. Am J Psychiatry (1998) 155(9):1288-90. doi:10.1176/ajp.155.9.1288

19. Roman-Urrestarazu A, Murray GK, Barnes A, Miettunen J, Jaaskelainen E, Maki P, et al. Brain structure in different psychosis risk groups in the Northern Finland 1986 birth cohort. Schizophr Res (2014) 153(1-3):143-9. doi:10.1016/j.schres.2013.12.019

\section{ACKNOWLEDGMENTS}

We would like to thank the participants and clinical research team at Larue D. Carter Memorial Hospital and the Indiana University Neuroscience Clinical Research Center for their support.

\section{FUNDING}

National Institute of Mental Health (R01 MH074983B, PI: WH).

20. Cahn W, Hulshoff Pol HE, Bongers M, Schnack HG, Mandl RC, Van Haren $\mathrm{NE}$, et al. Brain morphology in antipsychotic-naive schizophrenia: a study of multiple brain structures. Br J Psychiatry Suppl (2002) 43:s66-72. doi:10.1192/ bjp.181.43.s66

21. Supprian T, Ulmar G, Bauer M, Schuler M, Puschel K, Retz-Junginger P, et al. Cerebellar vermis area in schizophrenic patients - a post-mortem study. Schizophr Res (2000) 42(1):19-28. doi:10.1016/S0920-9964(99)00103-6

22. Bottmer C, Bachmann S, Pantel J, Essig M, Amann M, Schad LR, et al. Reduced cerebellar volume and neurological soft signs in first-episode schizophrenia. Psychiatry Res (2005) 140(3):239-50. doi:10.1016/j.pscychresns.2005.02.011

23. Kasparek T, Marecek R, Schwarz D, Prikryl R, Vanicek J, Mikl M, et al. Sourcebased morphometry of gray matter volume in men with first-episode schizophrenia. Hum Brain Mapp (2010) 31(2):300-10. doi:10.1002/hbm.20865

24. Rasser PE, Schall U, Peck G, Cohen M, Johnston P, Khoo K, et al. Cerebellar grey matter deficits in first-episode schizophrenia mapped using cortical pattern matching. Neuroimage (2010) 53(4):1175-80. doi:10.1016/j.neuroimage.2010.07.018

25. Ichimiya T, Okubo Y, Suhara T, Sudo Y. Reduced volume of the cerebellar vermis in neuroleptic-naive schizophrenia. Biol Psychiatry (2001) 49(1):20-7. doi:10.1016/S0006-3223(00)01081-7

26. Nopoulos PC, Ceilley JW, Gailis EA, Andreasen NC. An MRI study of cerebellar vermis morphology in patients with schizophrenia: evidence in support of the cognitive dysmetria concept. Biol Psychiatry (1999) 46(5):703-11. doi:10.1016/S0006-3223(99)00093-1

27. Wassink TH, Andreasen NC, Nopoulos P, Flaum M. Cerebellar morphology as a predictor of symptom and psychosocial outcome in schizophrenia. Biol Psychiatry (1999) 45(1):41-8. doi:10.1016/S0006-3223(98)00175-9

28. Potkin SG, Alva G, Fleming K, Anand R, Keator D, Carreon D, et al. A PET study of the pathophysiology of negative symptoms in schizophrenia. Positron emission tomography. Am J Psychiatry (2002) 159(2):227-37. doi:10.1176/ appi.ajp.159.2.227

29. Daum I, Schugens MM, Ackermann H, Lutzenberger W, Dichgans J, Birbaumer N. Classical conditioning after cerebellar lesions in humans. Behav Neurosci (1993) 107(5):748-56.

30. Topka H, Valls-Sole J, Massaquoi SG, Hallett M. Deficit in classical conditioning in patients with cerebellar degeneration. Brain (1993) 116(Pt 4):961-9. doi:10.1093/brain/116.4.961

31. Woodruff-Pak DS, Papka M, Ivry RB. Cerebellar involvement in eyeblink classical conditioning in humans. Neuropsychology (1996) 10(4):443-58. doi:10.1037/0894-4105.10.4.443

32. Kim JJ, Thompson RF. Cerebellar circuits and synaptic mechanisms involved in classical eyeblink conditioning. Trends Neurosci (1997) 20(4):177-81. doi:10.1016/S0166-2236(96)10081-3

33. Steinmetz JE. Brain substrates of classical eyeblink conditioning: a highly localized but also distributed system. Behav Brain Res (2000) 110(1-2):13-24. doi:10.1016/S0166-4328(99)00181-3

34. Christian KM, Thompson RF. Neural substrates of eyeblink conditioning: acquisition and retention. Learn Mem (2003) 10:427-55. doi:10.1101/ $\operatorname{lm} .59603$

35. Fanselow MS, Poulos AM. The neuroscience of mammalian associative learning. Annu Rev Psychol (2005) 56:207-34. doi:10.1146/annurev. psych.56.091103.070213

36. Brown SM, Kieffaber PD, Carroll CA, Vohs JL, Tracy JA, Shekhar A, et al. Eyeblink conditioning deficits indicate timing and cerebellar abnormalities in schizophrenia. Brain Cogn (2005) 58(1):94-108. doi:10.1016/j. bandc.2004.09.011 
37. Bolbecker AR, Hetrick WP, Johannesen JK, O’Donnell BF, Steinmetz JE, Shekhar AS. Secretin effects on cerebellar-dependent motor learning in schizophrenia. Am J Psychiatry (2009) 166(4):460-6. doi:10.1176/appi. ajp.2008.08040597

38. Bolbecker AR, Steinmetz AB, Mehta CS, Forsyth JK, Klaunig MJ, Lazar EK, et al. Exploration of cerebellar-dependent associative learning in schizophrenia: effects of varying and shifting interstimulus interval on eyeblink conditioning. Behav Neurosci (2011) 125(5):687-98. doi:10.1037/a0025150

39. Forsyth JK, Bolbecker AR, Mehta CS, Klaunig MJ, Steinmetz JE, O’Donnell BF, et al. Cerebellar-dependent eyeblink conditioning deficits in schizophrenia spectrum disorders. Schizophr Bull (2010) 38:751-9. doi:10.1093/schbul/ sbq148

40. Bolbecker AR, Mehta CS, Edwards CR, Steinmetz JE, O’Donnell BF, Hetrick WP. Eye-blink conditioning deficits indicate temporal processing abnormalities in schizophrenia. Schizophr Res (2009) 111(1-3):182-91. doi:10.1016/j. schres.2009.03.016

41. Gueorguieva R, Krystal JH. Move over ANOVA: progress in analyzing repeated-measures data and its reflection in papers published in the archives of general psychiatry. Arch Gen Psychiatry (2004) 61(3):310-7. doi:10.1001/ archpsyc.61.3.310

42. Bryk AS, Raudenbush SW. Heterogeneity of variance in experimental studies: a challenge to conventional interpretations. Psychol Bull (1988) 104(3):396-404. doi:10.1037/0033-2909.104.3.396

43. Raudenbush SW, Bryk AS. Hierarchical Linear Models: Applications and Data Analysis Methods. 2nd ed. (Vol. 1). Thousand Oaks, CA: Sage Publications, Inc (2002)

44. Bolbecker AR, Kent JS I, Petersen T, Klaunig MJ, Forsyth JK, Howell JM, et al. Impaired cerebellar-dependent eyeblink conditioning in first-degree relatives of individuals with schizophrenia. Schizophr Bull (2014) 40(5):1001-10. doi:10.1093/schbul/sbt112

45. Bolbecker AR, Westfall DR, Howell JM, Lackner R, Carroll CA, O’Donnell $\mathrm{BF}$, et al. Increased timing variability in schizophrenia and bipolar disorder. PLoS One (2014) 9(5):e97964. doi:10.1371/journal.pone.0097964

46. First MB, Spitzer RL, Gibbon M, Williams JBW. Structured Clinical Interview for DSM-IV-TR Axis I Disorders, Research Version, Patient Edition (SCID-I/P). New York: New York State Psychiatric Institute, Biometrics Research (2002).

47. First MB, Spitzer RL, Gibbon M, Williams JBW. Structured Clinical Interview for DSM-IV Axis I disorders - Nonpatient Version 2.0. New York, NY: Psychiatric Institute (1995).

48. Kay SR, Fiszbein A, Opler LA. The positive and negative syndrome scale (PANSS) for schizophrenia. Schizophr Bull (1987) 13(2):261-76. doi:10.1093/ schbul/13.2.261

49. Lang PJ, Greenwald MK. The International Affective Picture System Standardization Procedure and Initial Group Results for Affective Judgements: Technical Reports $1 A$ and $1 B$. Gainseville: Center for Research in Psychophysiology, University of Florida (1988).

50. Bolbecker AR, Mehta C, Johannesen JK, Edwards CR, O'Donnell BF, Shekhar A, et al. Eyeblink conditioning anomalies in bipolar disorder suggest cerebellar dysfunction. Bipolar Disord (2009) 11(1):19-32. doi:10.1111/j.1399-5618.2008.00642.x

51. Steinmetz AB, Edwards CR, Steinmetz JE, Hetrick WP. Comparison of auditory and visual conditioning stimuli in delay eyeblink conditioning in healthy young adults. Learn Behav (2009) 37(4):349-56. doi:10.3758/lb.37.4.349

52. Steinmetz AB, Skosnik PD, Edwards CR, Bolbecker AR, Steinmetz JE, Hetrick WP. Evaluation of bidirectional interstimulus interval (ISI) shift in auditory delay eye-blink conditioning in healthy humans. Learn Behav (2011) 39(4):358-70. doi:10.3758/s13420-011-0031-9

53. Pinheiro J, Bates D, DebRoy S, Sarkar D, R Core Team. NLME: Linear and Nonlinear Mixed Effects Models; R Package Version 3. (2009). Available from http://www.r-project.org/

54. Singer JD, Willett JB. Applied Longitudinal Data Analysis: Modeling Change and Event Occurrence. New York: Oxford (2003).

55. Parker KL, Andreasen NC, Liu D, Freeman JH, O’Leary DS. Eyeblink conditioning in unmedicated schizophrenia patients: a positron emission tomography study. Psychiatry Res (2013) 214(3):402-9. doi:10.1016/j. pscychresns.2013.07.006
56. Gould TJ, Steinmetz JE. Changes in rabbit cerebellar cortical and interpositus nucleus activity during acquisition, extinction, and backward classical eyelid conditioning. Neurobiol Learn Mem (1996) 65(1):17-34. doi:10.1006/ nlme.1996.0003

57. Mostofi A, Holtzman T, Grout AS, Yeo CH, Edgley SA. Electrophysiological localization of eyeblink-related microzones in rabbit cerebellar cortex. $J$ Neurosci (2010) 30(26):8920-34. doi:10.1523/jneurosci.6117-09.2010

58. Rosenfield ME, Moore JW. Connections to cerebellar cortex (Larsell's HVI) in the rabbit: a WGA-HRP study with implications for classical eyeblink conditioning. Behav Neurosci (1995) 109(6):1106-18. doi:10.1037/0735-7044.109.6.1106

59. Villarreal RP, Steinmetz JE. Neuroscience and learning: lessons from studying the involvement of a region of cerebellar cortex in eyeblink classical conditioning. J Exp Anal Behav (2005) 84(3):631-52. doi:10.1901/ jeab.2005.96-04

60. Freeman JH, Steinmetz AB. Neural circuitry and plasticity mechanisms underlying delay eyeblink conditioning. Learn Mem (2011) 18(10):666-77. doi:10.1101/lm.2023011

61. Gerwig M, Guberina H, Esser AC, Siebler M, Schoch B, Frings M, et al. Evaluation of multiple-session delay eyeblink conditioning comparing patients with focal cerebellar lesions and cerebellar degeneration. Behav Brain Res (2010) 212(2):143-51. doi:10.1016/j.bbr.2010.04.007

62. Edwards CR, Newman S, Bismark A, Skosnik PD, O’Donnell BF, Shekhar A, et al. Cerebellum volume and eyeblink conditioning in schizophrenia. Psychiatry Res (2008) 162(3):185-94. doi:10.1016/j.pscychresns.2007.06.001

63. Dimitrova A, Gerwig M, Brol B, Gizewski ER, Forsting M, Beck A, et al. Correlation of cerebellar volume with eyeblink conditioning in healthy subjects and in patients with cerebellar cortical degeneration. Brain Res (2008) 1198:73-84. doi:10.1016/j.brainres.2008.01.034

64. Carroll CA, O'Donnell BF, Shekhar A, Hetrick WP. Timing dysfunctions in schizophrenia as measured by a repetitive finger tapping task. Brain Cogn (2009) 71(3):345-53. doi:10.1016/j.bandc.2009.06.009

65. Carroll CA, Boggs J, O'Donnell BF, Shekhar A, Hetrick WP. Temporal processing dysfunction in schizophrenia. Brain Cogn (2008) 67(2):150-61. doi:10.1016/j.bandc.2007.12.005

66. Carroll CA, O'Donnell BF, Shekhar A, Hetrick WP. Timing dysfunctions in schizophrenia span from millisecond to several-second durations. Brain Cogn (2009) 70(2):181-90. doi:10.1016/j.bandc.2009.02.001

67. Lee SM, Chen L, Chow BK, Yung WH. Endogenous release and multiple actions of secretin in the rat cerebellum. Neuroscience (2005) 134(2):377-86. doi:10.1016/j.neuroscience.2005.04.009

68. Yung WH, Leung PS, Ng SS, Zhang J, Chan SC, Chow BK. Secretin facilitates GABA transmission in the cerebellum. J Neurosci (2001) 21(18):7063-8.

69. Fuchs JR, Robinson GM, Dean AM, Schoenberg HE, Williams MR, Morielli $\mathrm{AD}$, et al. Cerebellar secretin modulates eyeblink classical conditioning. Learn $\operatorname{Mem}$ (2014) 21(12):668-75. doi:10.1101/lm.035766.114

70. Williams MR, Fuchs JR, Green JT, Morielli AD. Cellular mechanisms and behavioral consequences of Kv1.2 regulation in the rat cerebellum. J Neurosci (2012) 32(27):9228-37. doi:10.1523/jneurosci.6504-11.2012

71. Demirtas-Tatlidede A, Freitas C, Cromer JR, Safar L, Ongur D, Stone WS, et al. Safety and proof of principle study of cerebellar vermal theta burst stimulation in refractory schizophrenia. Schizophr Res (2010) 124(1-3):91-100. doi:10.1016/j.schres.2010.08.015

Conflict of Interest Statement: The authors declare that the research was conducted in the absence of any commercial or financial relationships that could be construed as a potential conflict of interest.

Copyright (๑) 2016 Bolbecker, Petersen, Kent, Howell, O’Donnell and Hetrick. This is an open-access article distributed under the terms of the Creative Commons Attribution License (CC BY). The use, distribution or reproduction in other forums is permitted, provided the original author(s) or licensor are credited and that the original publication in this journal is cited, in accordance with accepted academic practice. No use, distribution or reproduction is permitted which does not comply with these terms. 